Published in final edited form as:

J Am Chem Soc. 2019 November 27; 141(47): 18668-18672. doi:10.1021/jacs.9b10875.

\title{
Enantioselective Olefin Hydrocyanation Without Cyanide
}

\author{
Alexander W. Schuppe ${ }^{\dagger}$, Gustavo M. Borrajo-Calleja ${ }^{\dagger}$, Stephen L. Buchwald \\ Department of Chemistry, Massachusetts Institute of Technology, 77 Massachusetts Avenue, \\ Cambridge, Massachusetts 02139, United States
}

\begin{abstract}
The enantioselective hydrocyanation of olefins represents a conceptually straightforward approach to prepare enantiomerically enriched nitriles. These, in turn, comprise or are intermediates in the synthesis of many pharmaceuticals and their synthetic derivatives. Herein, we report a cyanide-free dual $\mathrm{Pd} / \mathrm{CuH}$-catalyzed protocol for the asymmetric Markovnikov hydrocyanation of vinyl arenes and the anti-Markovnikov hydrocyanation of terminal olefins in which oxazoles function as nitrile equivalents. After an initial hydroarylation process, the oxazole substructure was deconstructed using a [4+2]/retro-[4+2] sequence to afford the enantioenriched nitrile product under mild reaction conditions.
\end{abstract}

\section{Graphical Abstract}

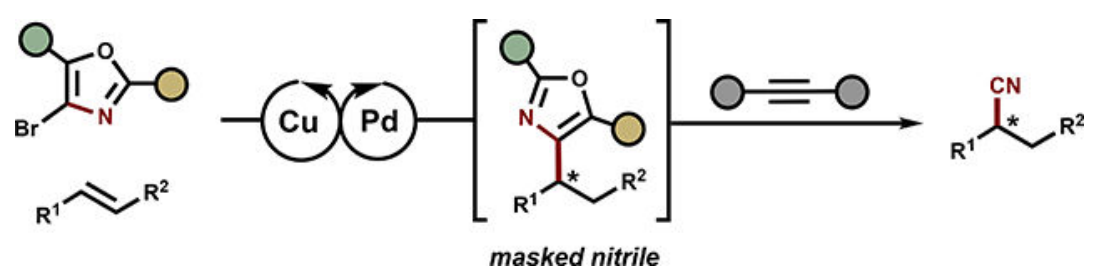

Nitriles are a ubiquitous class of compounds present in many pharmaceuticals, ${ }^{1}$ secondary metabolites, ${ }^{2}$ and polymers. ${ }^{3}$ Owing to their unique chemical reactivity, nitriles often serve as precursors to numerous additional important functional groups in organic synthesis, including $N$-heterocycles, carbonyl compounds, and amines. ${ }^{4}$ Although nitriles can be accessed by many methods, the conversion of olefins to alkyl nitriles via transition metalcatalyzed olefin hydrocyanation represents one of the most conceptually straightforward processes. While hydrocyanation of feedstock olefins is conducted on a million-metric ton scale annually to produce nitrile precursors to polymers, ${ }^{3}$ these protocols employ hydrogen cyanide and form almost exclusively achiral products. Despite the numerous improvements in the racemic hydrocyanation of olefin feedstocks ${ }^{5}$ and fine chemicals, ${ }^{6}$ the reaction

*Corresponding Author, sbuchwal@mit.edu.

$\dagger$ Author contributions

A.W.S. and G.M.B.-C. contributed equally.

The authors declare the following competing financial interest(s): MIT has obtained patents for some of the ligands that are described in this Communication from which S.L.B. and former/current co-workers receive royalty payments.

Supporting Information.

The Supporting Information is available free of charge on the ACS Publications website. Experimental procedures, and characterization data for all new compounds including ${ }^{1} \mathrm{H}$ - and ${ }^{13} \mathrm{C}-\mathrm{NMR}$ spectra, SFC and HPLC traces (PDF). 
conditions and substrates employed in the analogous asymmetric variant of this transformation have advanced minimally since the seminal work by Jackson ${ }^{7 \mathrm{a}}$ and RajanBabu. ${ }^{7 \mathrm{~b}-7 \mathrm{~d}, 7 \mathrm{~g}}$

Asymmetric olefin hydrocyanation is typically achieved through the formal addition of hydrogen cyanide, either generated in situ or employed directly in gaseous form, across an olefin facilitated by a chiral phosphine-ligated metal catalyst (Scheme 1A) ${ }^{7-8}$ Aside from the potential safety concerns of working with hydrogen cyanide, ${ }^{9}$ many of these asymmetric methods are limited to vinyl arenes and employ non-commercially available ligands. ${ }^{7,10-11}$ Alternative methods to access enantioenriched nitriles, including $\mathrm{C}-\mathrm{H}$ cyanation, ${ }^{12} \mathrm{a}$ arylation of prefunctionalized nitriles ${ }^{13}$ and enantioselective protonation of silyl ketene imines, ${ }^{14}$ have also been developed employing various precursors. ${ }^{15}$

Our continued interest in enantioselective alkene hydrofunctionalization reactions led us to envision the development of a catalytic protocol to access enantioenriched a-alkyl-aarylnitriles, represented by 3 (Figure 1B). ${ }^{16-18}$ We proposed that the critical $\mathrm{C}-\mathrm{CN}$ bond of the nitrile could be forged through an initial dual $\mathrm{Pd} / \mathrm{CuH}$-catalyzed asymmetric olefin hydroarylation ${ }^{17}$ reaction using a $N$-heterocyclic compound as a nitrile surrogate, thus obviating the need to employ cyanide either directly or transiently formed. A subsequent thermal-[4+2]/retro-[4+2] sequence with the appropriate dienophile could furnish the enantioenriched nitrile. However, at the outset, it was unclear to us which $N$-heterocycle would best serve as a masked nitrile, since pyrimidines, pyrazines, oxazoles and several other heterocycles have all been shown to expel nitriles as byproducts in cycloaddition reactions with alkynes. ${ }^{19-21}$ We reasoned that an oxazole, despite its limited precedent in forming nitriles, ${ }^{19}$ would be an ideal nitrile precursor for this transformation as it does not introduce any regiochemical complications and is an electron-rich aza-diene. ${ }^{21}$

Figure $1 \mathrm{C}$ details our proposed dual $\mathrm{Pd} / \mathrm{CuH}$ catalytic cycle for the aforementioned approach. Enantioselective hydrocupration of an olefinic substrate (1) by a CuH catalyst (I), generated in situ through the use of a $\mathrm{Cu}(\mathrm{I})$ salt, chiral phosphine ligand, and silane, would form an enantioenriched $\mathrm{Cu}(\mathrm{I})$ alkyl intermediate (II). Meanwhile, the Pd catalytic cycle would begin with oxidative addition of a ligated $\mathrm{Pd}(0)$ species (III) into a 2-halo-oxazole (2) forming complex IV. Stereospecific transmetallation of II with Pd species IV would result in an alkyl Pd(II) complex (V), which following reductive elimination furnishes an intermediate enantioenriched oxazole (4). The formed copper(I) halide (VI) could regenerate the active $\mathrm{CuH}$ catalyst after a $\sigma$-bond metathesis reaction in the presence of an appropriate base and silane. ${ }^{17-18}$ For this approach to be successful, the rates of both catalytic cycles would need to be well aligned to prevent any deleterious side pathways or the racemization of the alkyl copper species II. ${ }^{17}$ After this hydroarylation process, as depicted in Figure 1B, a subsequent thermal [4+2] cycloaddition between oxazole 4 and an alkyne would form a highly strained 7-oxa-2-azabicyclo[2.2.1]heptadiene derivative (5), and upon a retro-[4+2] cycloaddition the nitrile product is liberated along with an electron deficient furan (6). Thus, we reasoned that the judicious choice of a 2,5-disubstituted-4-halo-oxazole (2) coupling partner would be paramount to achieving both a highly enantioselective hydroarylation step and an efficient $[4+2] /$ retro-[4+2] sequence. 
Accordingly, we focused on finding a suitable halo-oxazole coupling partner (2) and a set of experimental reaction conditions for the asymmetric olefin hydrocyanation using styrene (1a) as a model substrate (Table 1). Our investigation of the optimal reaction conditions identified oxazole $\mathbf{2} \mathbf{a}$ as an excellent nitrile surrogate and the commercially available alkyne $\mathbf{7 a}$ as a suitable dienophile. When $\mathbf{2 a}$ and $\mathbf{7 a}$ were utilized in conjunction with $\left[\mathrm{Pd}(\right.$ cinnamyl $) \mathrm{Cl}_{2}$, BrettPhos (L3), P1, NaOTMS, and $\mathrm{Me}_{2}(\mathrm{Ph}) \mathrm{SiH}$, the desired nitrile 3a was formed in high yield and enantioselectivity (entry 1,96\% ${ }^{1} \mathrm{H}$ NMR yield and 97:3 er), without isolation of the alkyl oxazole intermediate (4). Evaluation of a series of $\mathrm{Cu}$ salts and chiral bisphosphines (entries 1-5) led us to discover the air-stable $\mathrm{Cu}(\mathrm{I})$ precatalyst $\mathbf{P 1}$, which enabled the reaction to be set up without the use of an inert-atmosphere glovebox. ${ }^{22}$ Use of the previously described (S)-DTBM-SEGPHOS-ligated CuCl precatalyst $\mathbf{P 2}^{18 \mathrm{~b}}$ formed the desired product in similar yield but with considerably lower enantioselectivity (entry 2). Variation of the biarylphosphine backbone (entries 6-7) or the absence of a Pdcatalyst (entry 8 ) resulted in diminished yield or no product formation respectively. Examination of an alternative to $\mathbf{2 a}$ as the nitrile surrogate highlighted the crucial role of the oxazole substituents in this transformation. Modification of the substituent at the 5-position from methyl to phenyl (2) delivered nitrile 3a in considerably lower yield and enantioselectivity, presumably due to the electron-poor nature of the corresponding alkyl oxazole intermediate (entry 9). While our previous reports on enantioselective olefin hydroarylation ${ }^{18 \mathrm{~b}}$ suggested that a 2 -chloro- $N$-heterocycle was more efficient in the hydrofunctionalization reaction than the corresponding hetereoaryl bromide, use of $\mathbf{2 c}$ in the current process resulted in minimal olefin hydrocyanation (entry 10). A variety of acetylene diester derivatives, such as the di- $n$-octyl substituted ester (7b), performed well as dienophiles. Notably, the judicious choice of dienophile coupling partner aided in the purification of the nitrile products (see below and the Supporting Information for details).

Having established appropriate reaction conditions for the asymmetric olefin hydrocyanation reaction, we investigated the scope of vinyl arene substrates (Scheme 1). Vinyl arenes bearing a substituent at the para-position, such as phenyl (3b), isobutyl (3d), or thiomethyl (3e), were well tolerated under the reaction conditions, resulting in good yields and enantioselectivity of the nitrile product. Facile enantiospecific hydrolysis could convert nitrile $3 \mathbf{d}$ and $\mathbf{3 g}$ to ibuprofen ${ }^{10}$ and cicloprofen, ${ }^{14}$ respectively, both of which are nonsteroidal anti-inflammatory drugs (NSAIDs). ${ }^{23} \mathrm{~A}$ vinyl arene containing orthosubstitution was effectively converted to the nitrile (3c) in high yield and enantiopurity. Moreover, substrates containing heterocycles, including benzofuran (3f), indoline (3h), $N$ tosyl-indole (3i), carbazole (3j), pyrazole (3k), morpholine (3m), and $N$-Boc-piperzine (3o), were smoothly transformed to the nitrile product with excellent selectivity. Additionally, 1,2disubstituted alkenes (Scheme 1B), a problematic substrate class for complementary Nicatalyzed asymmetric olefin hydrocyanation methods, ${ }^{7 \mathrm{~h}}$ performed well under our reaction conditions (3l and $\mathbf{3 m}$ ). However, cyclic olefins were difficult substrates for this transformation. Nitrile $\mathbf{3 n}$ was isolated in moderate yield and enantioselectivity when $\mathbf{1 n}$ was subjected to the standard catalytic system. We hypothesized that this diminished yield may reflect a slower rate of transmetallation between the proposed organometallic species II and IV, potentially due to a more sterically congested transition state, or a slower rate of hydrocupration of $\mathbf{1 n}$. To further highlight the applicability of this formal olefin 
hydrocyanation method to access medicinally relevant molecules, we synthesized an intermediate (3o) en route to $\mathbf{8}$, a USP28 inhibitor (Scheme 1C). Conversion of $\mathbf{3 o}$ to $\mathbf{8}$ could be achieved via reduction of the nitrile (3o) and acylation of the resulting primary amine. ${ }^{24}$

We were interested in extending this chemistry toward the anti-Markovnikov hydrocyanation of unactivated olefins. In line with our previous work, ${ }^{18 \mathrm{~b}}$ we anticipated the antiMarkovnikov hydrocyanation to be more challenging due to the higher hydrocupration barrier. ${ }^{25}$ However, we were able to perform the hydrocyanation of terminal olefins without significantly modifying the standard reaction conditions (Scheme 2). Overall, this process tolerates the presence of a variety of important structural elements $(\mathbf{1 0 a}-\mathbf{1 0 g})$, including an ester (10b), dioxolane (10c), benzothiazole (10e), indole (10f) and an amide (10g). Furthermore, the corresponding alkyl nitriles were isolated in high yield and regioselectivity. Hydrocyanation of terminal alkene (9d) accentuated the degree of chemoselectivity for this process, which generated $\mathbf{1 0 d}$ in good yield without any detectable hydrocyanation of the trisubstituted alkene. We further demonstrated the utility of this method by synthesizing the nitrile derivative (10g) of the cardiovascular drug Cilostazol (11), which could conceivably be converted to $\mathbf{1 1}$ following deprotection and tetrazole formation. ${ }^{26}$ As previously mentioned, reduction of the halo-oxazole (2) and the olefinic coupling partner represents potential side reactions for this transformation. Formation of a significant amount of reduced $\mathbf{9 g}$ was observed when the olefin was subjected to the standard reaction conditions. A decrease in the amount of $\mathbf{P 1}$ utilized, from 6.0 to $4.0 \mathrm{~mol} \%$, was necessary to improve the efficiency of the dual $\mathrm{CuH} / \mathrm{Pd}$ catalytic system and deliver amide $10 \mathrm{~g}$ as the major product.

Enantioenriched alkyl nitriles (3) often undergo epimerization or decomposition under a variety of acidic, basic and oxidative conditions, thus making further manipulation of the resulting nitrile product potentially challenging. ${ }^{7 f, 27}$ To obviate these degradation pathways, we envisioned that the chiral alkyl oxazole (4) may serve as a stable masked nitrile in multistep organic synthesis, which could be revealed at a later stage under neutral reaction conditions (Scheme 3). To illustrate this concept, we employed 1,2-disubstituted olefin 1p as a simple representative example. An initial asymmetric olefin hydroarylation reaction installed the oxazole substructure (4p), which was followed by silyl group removal, either under acid or fluoride-mediated conditions, and basic functionalization of the resulting phenol to yield oxazole 4p' without any erosion of the enantioselectivity. A subsequent thermal cycloaddition sequence with alkyne $\mathbf{7 b}$ revealed the nitrile $(\mathbf{3 p})$ with complete enantiospecificity. We believe that this strategy will be further applicable in more sophisticated contexts and numerous reaction manifolds that would otherwise result in decomposition of the nitrile substructure.

In summary, we have developed an asymmetric olefin hydrocyanation sequence that relies on an oxazole as surrogate for a nitrile, thus avoiding the use of any sources of cyanide in the reaction mixture. These reaction conditions developed were broadened to the antiMarkovnikov hydrocyanation of unactivated olefins. We anticipate that this strategy of employing an enantioenriched alkyl oxazole as a masked nitrile in multistep synthesis will find further utility in a variety of scenarios. 


\title{
Supplementary Material
}

Refer to Web version on PubMed Central for supplementary material.

\section{ACKNOWLEDGMENT}

\begin{abstract}
Research reported in this publication was supported by the Arnold and Mabel Beckman Foundation for a postdoctoral fellowship to A.W.S., the Swiss National Science Foundation (SNSF) for a postdoctoral fellowship (P2GEP2-181266) to G.M.B.-C. and the National Institutes of Health (R35-GM122483 and F32-GM131633 to A.W.S.). We thank the National Institutes of Health for a supplemental grant for the purchase of supercritical fluid chromatography (SFC) equipment (GM058160-17S1). We thank Sigma-Aldrich for the generous donation of XPhos and BrettPhos ligands. We are grateful to Drs. Richard Liu, Scott McCann, and Christine Nguyen for advice on the preparation of this manuscript.
\end{abstract}

\section{REFERENCES}

(1). Fleming FF; Yao L; Ravikumar PC; Funk L; Shook BC Nitrile-Containing Pharmaceuticals: Efficacious Roles of the Nitrile Pharmacophore. J. Med. Chem 2010, 53, 7902-7917. [PubMed: 20804202]

(2). Fleming FF Nitrile-containing natural products. Nat. Prod. Rep 1999, 16, 597-606.

(3). Tolman CA; McKinney RJ; Seidel WC; Druliner JD; Stevens WR Homogeneous Nickel-Catalyzed Olefin Hydrocyanation. Adv. Catal 1985, 33, 1-46.

(4). (a) The Chemistry of the Cyano Group; Rappoport Z, Ed.; Wiley: Weinheim, 1971.(b) Larock RC; Yao T Formation of Nitriles, Carboxylic Acids, and Derivatives by Oxidation, Substitution, and Addition In Comprehensive Organic Transformations, 3rd ed.; Wiley: Weinheim, 2018.

(5). RajanBabu TV Hydrocyanation of Alkenes and Alkynes. Org. React 2011, 75, 1-72.

(6). (a)For selected recent examples of racemic olefin hydrocyanation, see: Gasper B; Carreira EM. Mild Cobalt-Catalyzed Hydrocyanation of Olefins with Tosyl Cyanide. Angew. Chem. Int. Ed 2007, 46, 4519-4522.(b) de Greef M; Breit B Self-Assembled Bidentate Ligands for the NickelCatalyzed Hydrocyanation of Alkenes. Angew. Chem. Int. Ed 2009, 48, 551-554.(c) Fang X; Yu P; Morandi B Catalytically reversible alkene-nitrile interconversion through controllable transfer hydrocyanation. Science 2016, 351, 832-836. [PubMed: 26912891] (d) Bunia A; Berganer K; Studer A Cooperative Palladium/Lewis Acid-Catalyzed Transfer Hydrocyanation of Alkenes and Alkynes Using 1-Methylcyclohexa2,5-diene-1-carbonitrile. J. Am. Chem. Soc 2018, 140, 16353-16359. [PubMed: 30392374] (e) Orecchia P; Yuan W; Oestreich M Transfer Hydrocyanation of $\alpha-$ and $a, \beta$-Substituted Styrenes Catalyzed by Boron Lewis Acids. Angew. Chem. Int. Ed 2019, 58, 3579-3583.

(7). (a)For selected previous reports on asymmetric olefin hydrocyanation, see: Elmes PS; Jackson WJ. Asymmetric addition of hydrogen cyanide to alkenes catalyzed by a zerovalent palladium compound. J. Am. Chem. Soc 1979, 101, 6128-6129.(b) RajanBabu TV; Casalnuovo AL Tailored ligands for asymmetric catalysis: the hydrocyanation of vinyl arenes. J. Am. Chem. Soc 1992, 114, 6265-6266.(c) Casalnuovo AL; RajanBabu TV; Ayers TA; Warren TH Ligand Electronic Effects in Asymmetric Catalysis: Enhanced Enantioselectivity in the Asymmetric Hydrocyanation of Vinylarenes. J. Am. Chem. Soc 1994, 116, 9869-9882.(d) RajanBabu TV; Casalnuovo AL Role of Electronic Asymmetry in the Design of New Ligands: The Asymmetric Hydrocyanation Reaction. J. Am. Chem. Soc 1994, 118, 6325-6326.(e) Yan M; Xu Q-Y; Chan AS C. Tetrahedron: Asymmetry 2000, 11, 845-849.(f) Wilting J; Janssen M; Müller C; Vogt D The Enantioselective Step in the Nickel-Catalyzed Hydrocyanation of 1,3-cyclohexadiene. J. Am. Chem. Soc 2006, 128, 11374-11375. [PubMed: 16939259] (g) Saha B; RajanBabu TV Nickel(0)-Catalyzed Asymmetric Hydrocyanation of 1,3-Dienes. Org. Lett 2006, 8, 4657-4659. [PubMed: 16986974] (h) Falk A; Göderz A-L; Schmalz H-G Enantioselective Nickel-Catalyzed Hydrocyanation of Vinylarenes Using Chiral Phosphine-Phosphite Ligands and TMS-CN as a Source of HCN. Angew. Chem. Int. Ed 2013, 52, 1576-1580.(i) Falk A; Cavalieri A; Nichol GS; Vogt D; Schmalz H-G Enantioselective nickel-catalyzed hydrocyanation using chiral phosphinephosphite ligands: recent improvements and insights. Adv. Synth. Catal 2015, 357, 3317-3320. 
(8). (a)For general reviews on asymmetric cyanation reactions, see: Khan N-U; Kureshy RI; Abdi SHR; Agrawal S; Jasra RV Metal catalyzed asymmetric cyanation reactions. Coord. Chem. Rev 2008, 252, 593-623.(b) Kurono N; Ohkuma T Catalytic Asymmetric Cyanation Reactions. ACS Catal. 2016, 6, 989-1023.

(9). Romeder G Hydrogen Cyanide. e-EROS Encyclopedia of Reagents for Organic Synthesis. 2001.

(10). For a recent example of formal asymmetric olefin hydrocyanation, see: Li X; Yang J; Li S; Zhang D; Lv H; Zhang X Asymmetric Hydrocyanation of Alkenes without HCN. Angew. Chem. Int. Ed 2019, 58, 10828-10931.

(11). In the process of preparing our manuscript a complementary electrochemical approach to asymmetric olefin hydrocyanation was described, see: Lu S; Fu N; Ernst BG; Lee W-H; Frederick MO; DiStasio RA; Lin S Dual Electrocatalysis Enabled Enantioselective Hydrocyanation of Conjugated Alkenes. ChemRxiv Preprint, 2019, DOI: 10.26434/chemrxiv. 9784625.v1.

(12). Zhang W; Wang F; McCann SD; Wang D; Chen P; Stahl SS; Liu G Enantioselective cyanation of benzylic C__H bonds via copper-catalyzed radical relay. Science 2016, 353, 1014-1018. [PubMed: 27701109]

(13). (a) Choi J; Fu GC Catalytic Asymmetric Synthesis of Secondary Nitriles via Stereoconvergent Negishi Arylations and Alkenylation of Racemic a-Bromonitriles. J. Am. Chem. Soc 2012, 134, 9102-9105. [PubMed: 22612264] (b) Kadunce NT; Reisman SE Nickel-Catalyzed Asymmetric Reductive Cross-Coupling between Heteroaryl Iodides and a-Chloronitriles. J. Am. Chem. Soc 2015, 137, 10480-10483. [PubMed: 26256474] (c) Jiao Z; Chee KC; Zhou J PalladiumCatalyzed Asymmetric a-Arylation of Alkylnitriles. J. Am. Chem. Soc 2016, 138, 16240-16243. [PubMed: 27936644]

(14). Guin J; Varseev G; List B Catalytic Asymmetric Protonation of Silyl Ketene Imines. J. Am. Chem. Soc 2013, 135, 2100-2103. [PubMed: 23362931]

(15). (a)For selected additional indirect approaches to access enantioenriched nitriles, see: Enders D; Plant A; Backhaus D; Reinhold. Asymmetric synthesis of a-substituted nitriles and cyanohydrins by oxidative cleavage of chiral aldehyde hydrazones with magnesium monoperoxyphthalate. Tetrahedron 1995, 51, 10699-10714.(b) Betke T; Rommelmann P; Oike K; Asano Y; Gröger H Cyanide-Free and Broadly Applicable Enantioselective Synthetic Platform for Chiral Nitriles through a Biocatalytic Approach. Angew. Chem. Int. Ed. 2017, 56, 12361-12366.(c) Wang D; Zhu N; Chen P; Lin Z; Liu G Enantioselective Decarboxylation Cyanation Employing Cooperative Photoredox Catalysis and Copper Catalysis. J. Am. Chem. Soc. 2017, 139, 1563215635. [PubMed: 29039930]

(16). Pirnot MT; Wang Y-M; Buchwald SL Copper Hydride Catalyzed Hydroamination of Alkenes and Alkynes. Angew. Chem. Int. Ed. 2016, 55, 48-57.

(17). Friis SD; Pirnot MT; Buchwald SL Asymmetric Hydroarylation of Vinylarenes Using a Synergistic Combination of CuH and Pd Catalysis. J. Am. Chem. Soc. 2016, 138, 8372-8375. [PubMed: 27346525]

(18). (a)For examples of racemic $\mathrm{CuH} / \mathrm{Pd}$ metal-catalyzed hydroarylation reactions, see Semba K; Ariyama K; Zheng H; Kameyama R; Sakaki S; Nakao Y Reductive Cross-Coupling of Conjugated Arylalkenes and Aryl Bromides with Hydrosilanes by Cooperative Palladium/Copper Catalysis. Angew. Chem. Int. Ed. 2016, 55, 6275-6279.(b) Friis SD; Pirnot MT; Dupuis LN; Buchwald SL A Dual Palladium and Copper Hydride Catalyzed Approach for Alkyl-Aryl CrossCoupling of Aryl Halides and Olefins. Angew. Chem. Int. Ed 2017, 56, 7242-7246.

(19). (a) Grigg R; Hayes R; Jackson JL Thermal Elimination Reactions of Nitrogen and Sulphur Heterocycles. J. Chem. Soc. D, 1969, 1167-1168.(b) Grigg R; Jackson JL Elimination of Nitriles in Retro-diene Reactions. J. Chem. Soc C 1970, 552-556.(c) Toshikazu I; Shuji N; Hiroyuki N; Jiro T; Yasushi I The Diels-Alder Reactions of 2-Alkyl-5-methoxy-4-(p-nitrophenyl)oxazoles with Ethylenic, Acetylenic, and Azo-Type Dienophiles. Bull. Chem. Soc. Jpn 1986, 59, 433-437.

(20). (a) Turchi IJ; Dewar MJS The Chemistry of Oxazoles. Chem. Rev 1975, 75, 389-437.(b) Jouano L-A; Renault K; Sabot C; Renard P-Y 5-Alkoxyoxazole-A Versatile Building Block in (Bio)organic Synthesis. Eur. J. Org. Chem 2016, 3264-3281.

(21). Boger DL Diels-Alder reactions of heterocyclic aza dienes. Scope and applications. Chem. Rev 1986, 86, 781-793. 
(22). Employing P1 outside the glovebox resulted in a similar yield and selectivity, see supporting information for details on benchtop reaction setup.

(23). Harrington PJ; Lodewijk E Twenty Years of Naproxen Technology. Org. Process Res. Dev 1997, $1,72-76$.

(24). Gerin DJ; Bair KW; Ioannidis S; Lancia DR; Li H; Mischke S; Ng PY; Richard D; Schiller SER; Shelekhin T; Wang Z Thienopyridine Carboxamides as Ubiquitin-Specific Protease Inhibitors. International Patent WO 139778 (A1), Feb. 13, 2017.

(25). (a) Lu G; Liu RY; Yang Y; Fang C; Lambrecht DS; Buchwald SL; Liu P Ligand-Substrate Dispersion Facilitates the Copper-Catalyzed Hydroamination of Unactivated Olefins. J. Am. Chem. Soc. 2017, 137, 16548-16555.(b) Thomas AA; Speck K; Kevlishvili I; Lu Z; Liu P; Buchwald SL Mechanistically Guided Design of Ligands That Significantly Improve the Efficiency of CuH-Catalyzed Hydroamination Reactions. J. Am. Chem. Soc. 2018, 140, 1397613984. [PubMed: 30244567]

(26). Neochoritis CG; Zhao T; Dömling A Tetrazoles via Multicomponent Reactions. Chem. Rev. 2019, 119, 1970-2042. [PubMed: 30707567]

(27). In some substrates ( $3 \mathrm{~b}$ and $3 \mathrm{e}$ ) epimerization of the nitrile product was observed upon exposure to silica flash column chromatography, which could be avoided by altering the method of purification, see supporting information for details. 
A. Prototypical Asymmetric Olefin Hydrocyanation

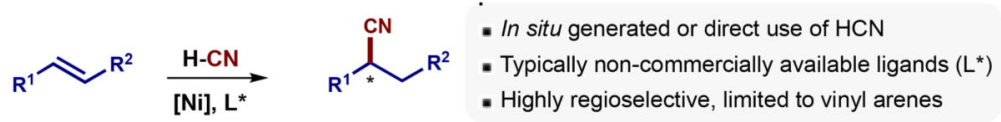

B. This Approach
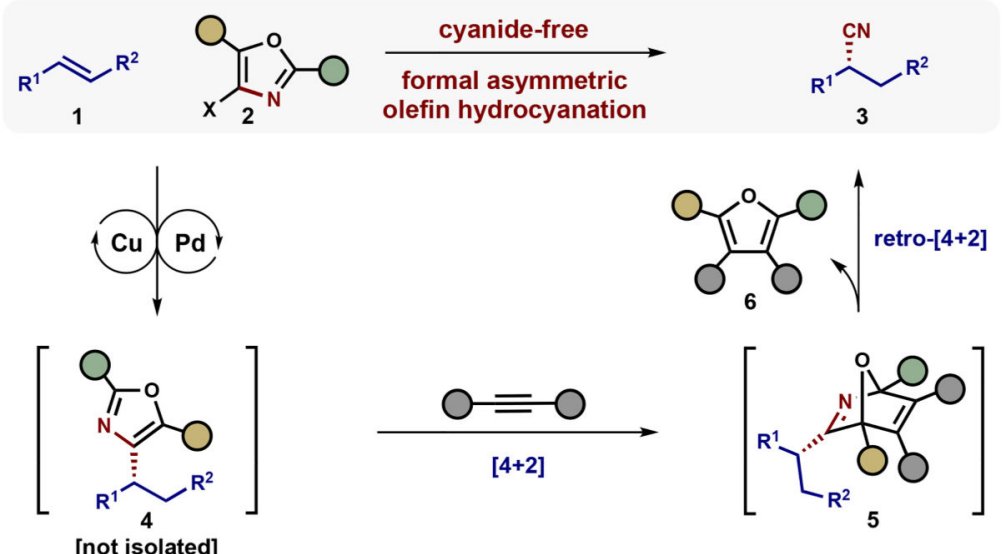

C. Proposed Dual Catalytic Cycle

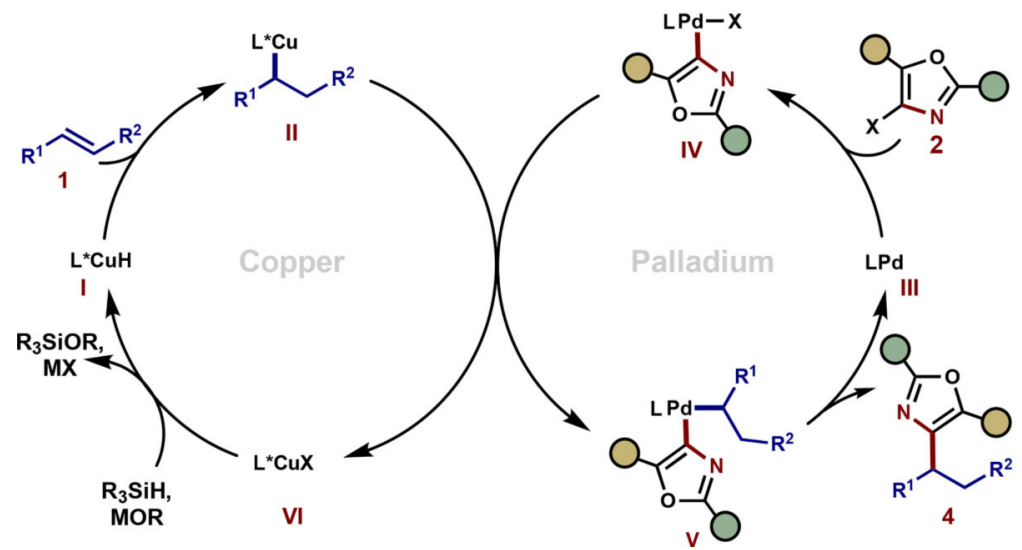

Figure 1.

A. Traditional approaches to asymmetric olefin hydrocyanation. B. Our dual Pd/CuHcatalyzed asymmetric olefin hydrocyanation using oxazoles as masked nitriles, followed by a thermal deconstruction of $\mathbf{4}$ to the enantioenriched nitrile. C. Proposed dual $\mathrm{Pd} / \mathrm{CuH}$ catalytic cycles for the hydrofunctionalization process involving a 2-halo-oxazole (2). 


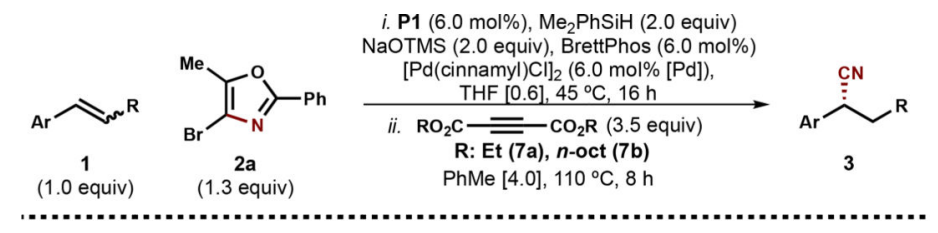

A. Vinyl (hetero)arenes
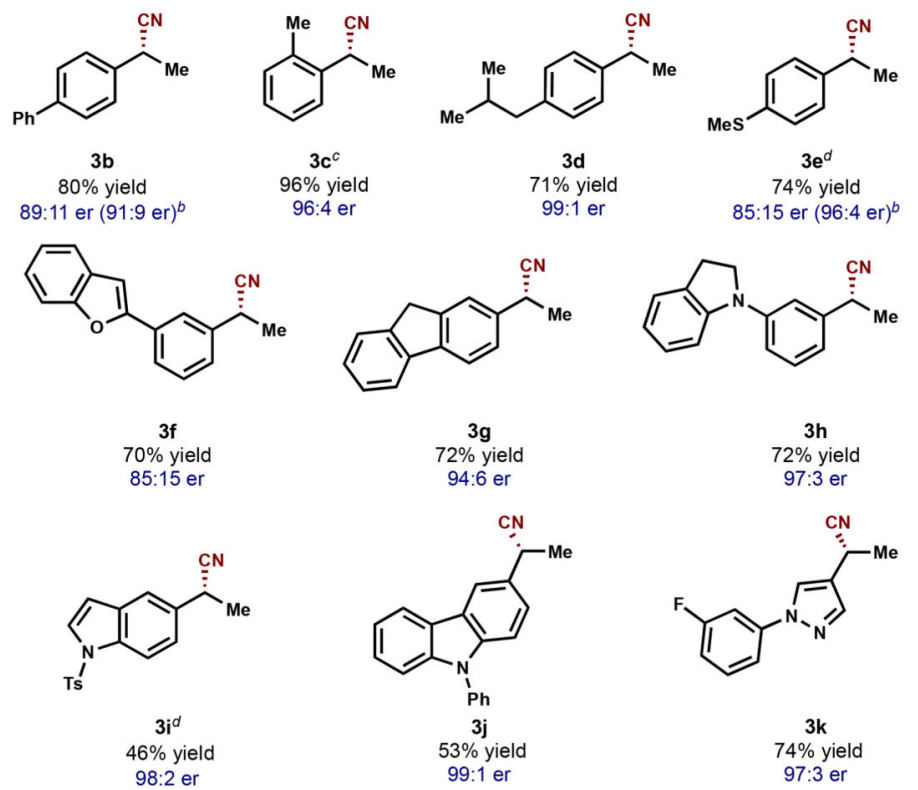

B. 1,2-disubstituted alkenes
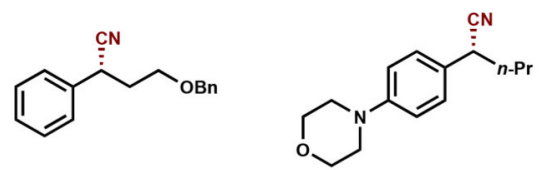<smiles>Cc1ccccc1COc1cccc2c1CCC[C@H]2C</smiles>
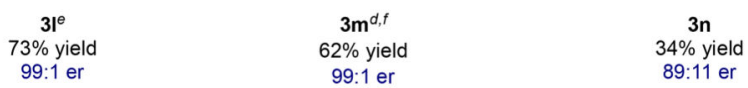

C. Synthesis of pharmaceutical intermediate

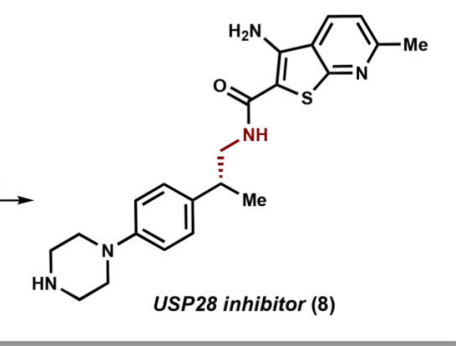

Scheme 1.

Substrate scope of the asymmetric Markovnikov hydrocyanation of vinyl arenes. ${ }^{a}$ ${ }^{a}$ All yields represent the average of isolated yields from two runs purified by silica flash chromatography with $0.5 \mathrm{mmol}$ alkene; alkyne (7b) was used unless otherwise noted, enantioselectivity determined by chiral SFC or HPLC. ${ }^{b}$ Alternative purification was used, see supporting information for details. ${ }^{C}$ Yield was determined by ${ }^{1} \mathrm{H}$ NMR spectroscopy using 1,1,2,2-tetrachloroethane as an internal standard due to the volatility of the product. 
${ }^{d}$ Alkyne (7a) was used. ${ }^{e}$ Intermediate oxazole $4 \mathbf{l}$ was purified, isolated yield reported over two steps. ${ }^{f} 1.5$ equiv of $\mathbf{2 a}$ and $24 \mathrm{~h}$ at $45^{\circ} \mathrm{C}$ 

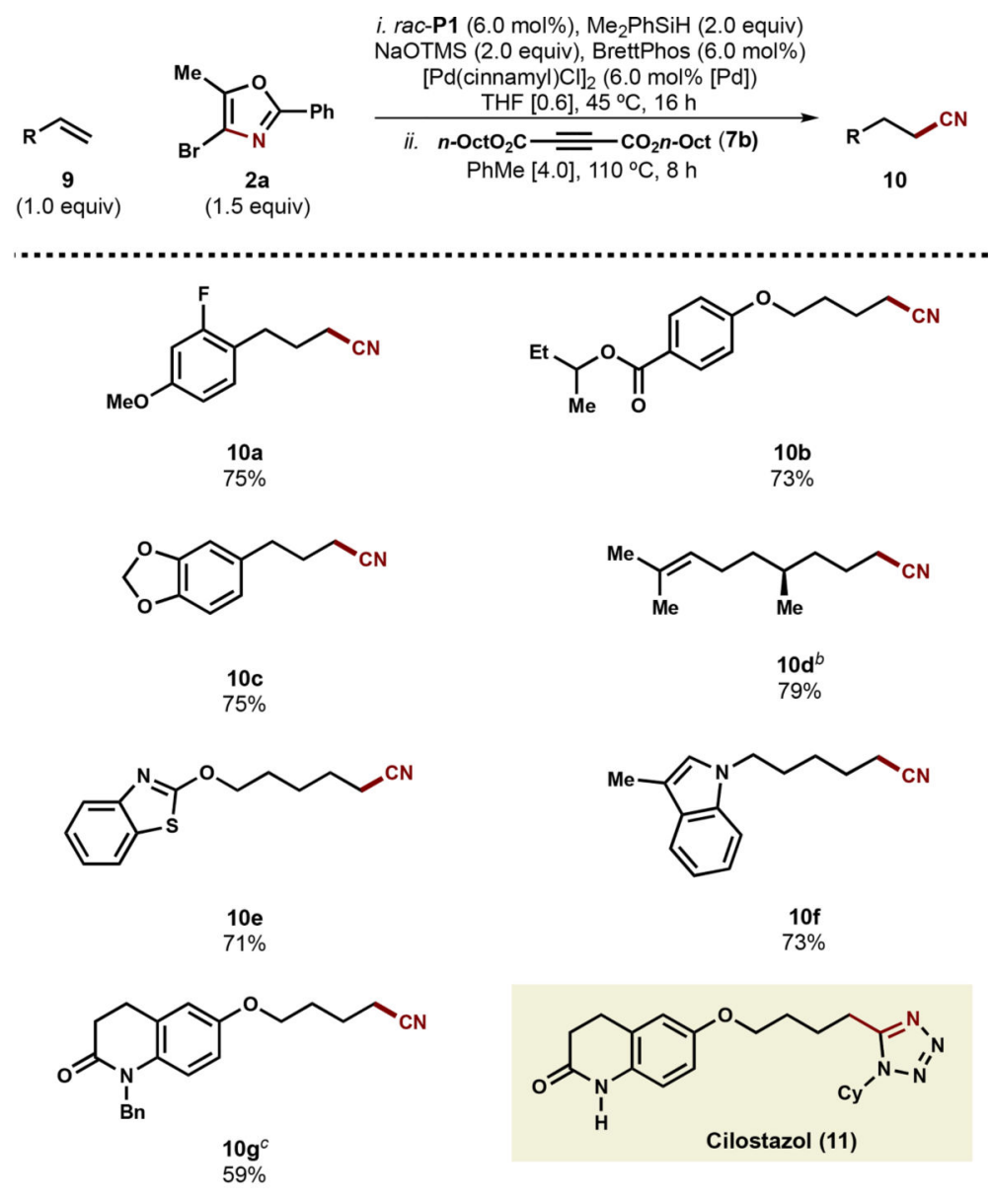

Scheme 2.

Substrate scope for the anti-Markovnikov hydrocyanation of unactivated olefins. ${ }^{a}$

${ }^{a}$ All yields represent the average of isolated yields from two runs purified by silica flash chromatography with $0.5 \mathrm{mmol}$ alkene. ${ }^{b}$ Yield was determined by ${ }^{1} \mathrm{H}$ NMR spectroscopy using 1,1,2,2-tetrachloroethane as an internal standard ${ }^{c} 4.0 \mathrm{~mol} \% \mathbf{P 1}$ 


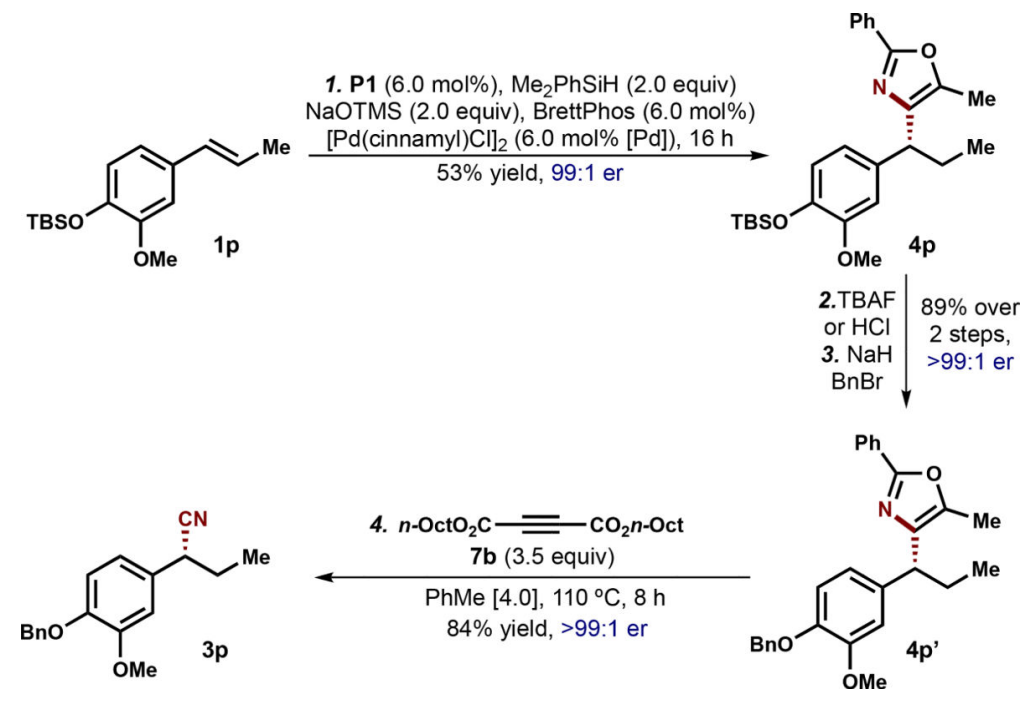

Scheme 3.

Enantioenriched oxazoles as masked nitriles in multistep synthesis

${ }^{a}$ Reagents and conditions: (1) Average of isolated yields from two runs on $0.5 \mathrm{mmol}$ scale;

(2) TBAF (1.5 equiv), THF [0.45], rt, 1.5 h or aq. $6 \mathrm{M} \mathrm{HCl} \mathrm{(5.0} \mathrm{equiv),} \mathrm{THF} \mathrm{[0.5],} \mathrm{rt,} 16$ h;

(3) $\mathrm{NaH}$ (1.2 equiv), $\mathrm{BnBr}$ (1.2 equiv), rt, $18 \mathrm{~h}, 89 \%$ or $81 \%$ over two steps, respectively $>99: 1$ er; (4) $7 \mathbf{b}$ (3.5 equiv), PhMe [4.0], $110^{\circ} \mathrm{C}, 8$ h, 84\%, >99:1 er 
Table 1.

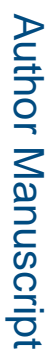

Optimization of the enantioselective hydrocyanation of styrene (1a). ${ }^{a}$

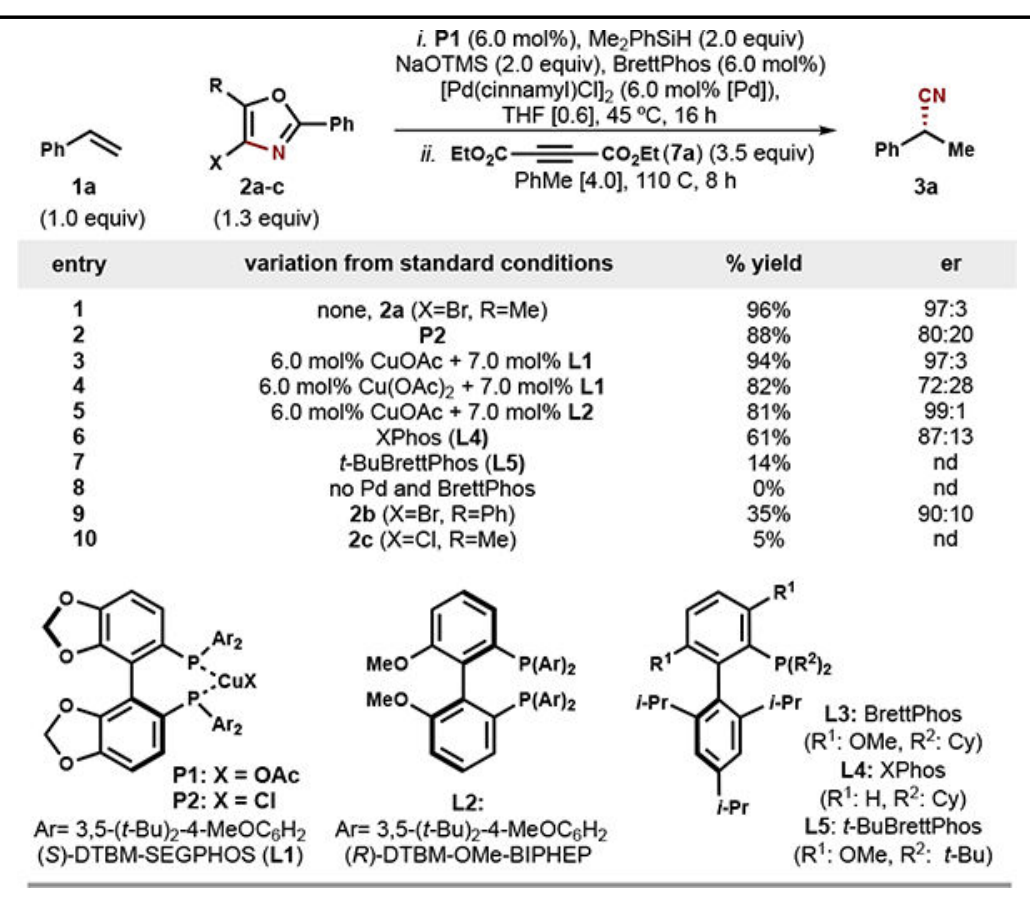

${ }^{a}$ Reaction conditions: $0.2 \mathrm{mmol}$ styrene (1.0 equiv), yields were determined by ${ }^{1} \mathrm{H}$ NMR spectroscopy of the crude reaction mixture, using $1,1,2,2$ tetrachloroethane as internal standard. Enantiomeric ratio (er) was determined by chiral SFC. nd: not determined. 